\title{
Positivity issues for the pinch-technique gluon propagator and their resolution
}

\author{
John M. Cornwall* \\ Department of Physics and Astronomy, University of California, Los Angeles CA 90095
}

\begin{abstract}
Although gauge-boson propagators in asymptotically-free gauge theories satisfy a dispersion relation, they do not satisfy the Källen-Lehmann (K-L) representation because the spectral function changes sign. We argue that this is a simple consequence of asymptotic freedom. On the basis of the QED-like Ward identities of the pinch technique (PT) we claim that the product of the coupling $g^{2}$ and the scalar part $\hat{d}\left(q^{2}\right)$ of the PT propagator, which is both gauge-invariant and renormalizationgroup invariant, can be factored into the product of the running charge $\bar{g}^{2}\left(q^{2}\right)$ and a term $\hat{H}\left(q^{2}\right)$ both of which satisfy the K-L representation although their product does not. We show that this behavior is consistent with some simple analytic models that mimic the gauge-invariant PT Schwinger-Dyson equations (SDE), provided that the dynamic gauge-boson mass is sufficiently large. The PT SDEs do not depend directly on the PT propagator through $\hat{d}$ but only through $\hat{H}$.
\end{abstract}

PACS numbers: 11.15.Tk, 11.15.Kc

$\mathrm{UCLA} / 09 / \mathrm{TEP} / 42$

\section{INTRODUCTION}

\section{A. Positivity and gluon mass}

A long-standing problem of non-Abelian gauge theories (NAGTs) is the lack of positivity of the imaginary part of the gauge-boson propagator, violating the Källen-Lehmann (K-L) representation. This was first pointed out in an early paper on the gauge-invariant pinch-technique (PT) propagator [1]. Later, many authors found the same behavior in lattice simulations of the gauge-dependent and unphysical propagator of the Landau gauge 2, 3, 4] (and references therein). Although it is questionable to assign a physical meaning to this lack of positivity in a gaugedependent quantity such as the Landau-gauge propagator, many authors see it as a sign of confinement, since the propagator of an unconfined field presumably has a normal K-L representation. Aubin and Ogilvie [3] trace it to technical deficiencies in lattice gauge-fixing procedures.

In this paper we argue that it is plausible (but unproven) that this lack of positivity is an elementary consequence of asymptotic freedom, and is simply resolved by a factorization of the PT propagator into two terms each of which is both gauge- and renormalization group (RG)-invariant, and each satisfies the K-L representation. We construct some simple analytic models of the PT propagator and vertex that illustrate the necessary positivity and absence of unphysical singularities, provided that there is a sufficiently-large dynamical gluon mass. We also discuss models of the Analytic Perturbation Theory (APT) type, which can satisfy positivity with zero gluon mass but still have unphysical behavior.

Section IA briefly covers notation as well as some background on the PT. In Section IIB we argue that the lack of positivity is a simple consequence of asymptotic freedom and the fact [1] that the product of the coupling $g^{2}$ and the (scalar part of the) gauge-invariant PT propagator $\hat{d}\left(q^{2}\right)$ is not only gauge-invariant but also RG-invariant, independent of the choice of a renormalization point. This makes this product a truly physical quantity. The same is true for the photon propagator in QED, as has been known for decades, and for the same reason: The Ward identities of QED or of the PT require that the gluon vertex function renormalization constant and a wave-function renormalization constant be the same.

Not every non-perturbative approximation for the propagator can be expected to satisfy these positivity constraints, in an asymptotically-free gauge theory. In the rest of the paper we construct non-perturbative models that do satisfy them, provided that there is a large enough dynamical gluon mass.

Section [IC is an illustration, within the context of an analytically-soluble model similar to an earlier [1] onedressed-loop Schwinger-Dyson equation (SDE) for the PT propagator, of how the positivity argument above can only be realized with a sufficiently large gluon mass. In the model, provided that the gluon mass $m$ is large enough (on the QCD scale $\Lambda$ ) each factor in the product $\bar{g}^{2} \bar{H}$ behaves precisely as would be expected, with no bizarre behavior coming from non-positivity. But if $m / \Lambda$ is less than a critical value $m_{c} / \Lambda$ spurious singularities arise, such as ghost or spacelike poles in the propagator. We estimate $m_{c} / \Lambda \simeq 1.2$ in our study of the one-dressed-loop PT propagator; given the approximations made there, we believe the range should be from 1 to 1.5 or so. This effectively provides,

\footnotetext{
*Email cornwall@physics.ucla.edu
} 
as we will see, an upper limit to the running charge at zero momentum: $\alpha_{s}(0) \equiv \bar{g}^{2}(0) / 4 \pi \leq 0.5-0.7$. This is fairly consistent with other determinations from phenomenology [5], studies [1, 6, 7, 8] of the PT SDE, and a study of the functional Schrödinger equation [9].

Section [II] briefly reviews the evolution of APT from an originally massless form [10] with correct positivity properties, yet showing unphysical behavior, to a massive form rather similar to that of [1] and the present paper. In the original APT positivity was satisfied, although the PT upper limit on $\alpha_{s}(0)$ is exceeded, with $\alpha_{s}(0) \approx 1 /(4 \pi b) \approx 1.1$. This in itself is not necessarily serious, but what is serious is that the APT running charge, even though finite at zero momentum, has infinite slope. A later work [11] corrects this deficiency by invoking an ad hoc gluon mass, in somewhat the same spirit as the PT gluon mass, with results quite similar to our first model. Other authors [12] have also invoked masses as cutoffs for APT.

Section IV remarks on the important fact that the vertex SDE can be reformulated entirely in terms of the propagator factor $\hat{H}$ with a positive spectral function and a special half-proper vertex that is both gauge- and RGinvariant; the original PT propagator $\hat{d}$, with its positivity violations, never appears. This reformulation avoids possible violations of positivity that could allow unphysical vertex behavior. We illustrate with an analytic approximation inspired by a one-dressed-loop toy model [13] of the Schwinger-Dyson equation for the three-gluon vertex and show that it has a spurious spacelike singularity if the gluon mass is too small.

Section IVC is a discussion of certain typical all-order extensions and resummations of the massless toy model that still lead to unphysical singularities; these can only be resolved with a dynamical gluon mass.

\section{B. The positivity problem and asymptotic freedom}

Write the obvious factorization of the product $g^{2} \hat{d}$ :

$$
g^{2} \hat{d}\left(q^{2}\right)=\bar{g}^{2}\left(q^{2}\right) \hat{H}\left(q^{2}\right)
$$

where $\bar{g}\left(q^{2}\right)$ is the gauge-, scheme-, and renormalization-point-independent running charge of the PT. The other factor $\hat{H}\left(q^{2}\right)$ has the same properties, since the product does. We argue that both factors obey a standard K-L representation with a positive imaginary part (our metric is such that $q^{2}>0$ for timelike vectors):

$$
\begin{aligned}
\bar{g}^{2}\left(q^{2}\right) & =\frac{1}{\pi} \int_{4 m^{2}}^{\infty} d \sigma \frac{\rho(\sigma)}{\sigma-q^{2}-i \epsilon} \\
\hat{H}\left(q^{2}\right) & =\frac{1}{\pi} \int_{4 m^{2}}^{\infty} d \sigma \frac{\rho_{H}(\sigma)}{\sigma-q^{2}-i \epsilon}
\end{aligned}
$$

and $\rho, \rho_{H}$ are positive. The lower limit involves the dynamical gluon mass $m$, which we discuss later.

We plausibly know the behavior at infinite momentum of both factors in the product. Asymptotic freedom tells us that

$$
\bar{g}\left(q^{2}\right) \rightarrow_{q^{2} \rightarrow \infty} \frac{1}{b \ln \left(-q^{2} / \Lambda^{2}\right)} .
$$

where $b$ is the lowest-order coefficient in the beta-function and $\Lambda$ is the QCD scale. As for $\hat{H}\left(q^{2}\right)$ there is no reason from perturbation theory or non-perturbative PT constructions to believe that it departs from the simple free-field behavior $1 / q^{2}$ for large momentum. For example, it is well-known that the PT is equivalent order by order to the background field Feynman gauge [14], and old perturbative calculations in this gauge through two loops [15] show that all large-momentum logarithms are accounted for in the running charge. The result is that at large momentum the PT propagator vanishes according to $\hat{d}\left(q^{2}\right) \sim 1 /\left(q^{2} \ln q^{2}\right)$. But a propagator vanishing more rapidly than $1 / q^{2}$ implies that the spectral function in the would-be $\mathrm{K}-\mathrm{L}$ representation is necessarily negative somewhere. There is, we claim, only an indirect connection - at least for the gauge-invariant PT propagator-between non-positivity and confinement (a connection only to the extent that asymptotic freedom implies confinement). In fact, the gluon is not confined, but screened, in the usual sense that the string in the adjoint-representation Wilson loop always breaks at sufficiently large distance even with no adjoint matter fields. We will not attempt any analysis of non-positivity in the Landau-gauge, but it is likely that even if some non-positivity comes from the Aubin-Ogilvie [3] effect, there will still be some residual non-positivity coming from asymptotic freedom.

What happens at infrared momenta? It has long been argued [1, 16] that the QCD gluon should pick up a dynamical mass that completely preserves local gauge symmetry. This is consistent with phenomenology (for example, [5], 17]), and a number of studies of the PT Schwinger-Dyson equations [18] have found [1, 6, 7, 8] a PT pole mass $m$ of order $0.6 \mathrm{GeV}$. These studies also indicate that the mass runs with momentum and should be denoted $m\left(q^{2}\right)$, consistent 
with the operator-product expansion result that $m^{2}\left(q^{2}\right)$ vanishes (modulo logarithms) like $\left\langle G_{\mu \nu} G^{\mu \nu}\right\rangle / q^{2}$ at large momentum 19. There is a large body of lattice-simulation evidence in the Landau gauge [20, 21, 22, 23, 24, 25, 26, 27, 28, 29, 30, 31, 32, 33, 34, 35, 36, 37, 38] for a gluon mass of several hundred MeV. (The pole mass of the gluon propagator in any gauge is gauge-invariant and physical, although because it is a timelike pole it is not easy to determine from lattices simulations.) We think it plausible, then, that $\hat{H}\left(q^{2}\right)$ has some such form as:

$$
\hat{H}\left(q^{2}\right)=\frac{1}{\hat{m}^{2}\left(q^{2}\right)-q^{2}-i \epsilon}
$$

where $m\left(q^{2}\right)$ is the running mass. For simplicity and brevity, we use in this paper a fixed gluon mass; running does not interfere with the main positivity arguments. Then $\hat{H}$ is a simple free massive propagator.

\section{A MODEL OF THE PT INVERSE PROPAGATOR}

\section{A. A few words on the pinch technique}

Begin with some notation. The pinch-technique propagator has the form:

$$
\hat{\Delta}_{\alpha \beta}(q)=P_{\alpha \beta}(q) \hat{d}(q)+\xi \frac{q_{\alpha} q_{\beta}}{q^{4}} ; \quad P_{\alpha \beta}(q)=-g_{\alpha \beta}+\frac{q_{\alpha} q_{\beta}}{q^{2}} .
$$

The corresponding inverse pinch-technique propagator is:

$$
\hat{\Delta}_{\alpha \beta}^{-1}(q)=P_{\alpha \beta}(q)\left[q^{2}+\hat{\Pi}(q)\right]+\frac{1}{\xi} q_{\alpha} q_{\beta} .
$$

The scalar function $\hat{d}$ is completely independent of the gauge chosen.

The PT is a systematic way of extracting gauge-invariant proper self-energies, vertices, and the like from gaugeinvariant quantities such as the S-matrix. The PT propagator, for example, is not constructed just from the usual Feynman graphs; it also receives contributions from other graphs through a so-called "pinch", in which longitudinal momenta in numerators, coming from vertices and propagators, trigger Ward identities leading to the replacement of certain propagators by unity. This changes the topology of the graphs where this occurs, and some of these pinch contributions are readily recognized as contributions of propagator type. Although not recognized at the beginning, it is now known [14] that the PT is the same graphical expansion as that of the background field-Feynman gauge. Because the whole point of the PT is to maintain gauge invariance, it is essential that, when this graphical expansion is resummed to a dressed-loop or skeleton expansion, all Green's functions appearing in the skeleton expansion obey the correct Ward identities. In the case of the PT these are the naive Ward identities of QED, with no ghosts.

It would appear that progress can only be made by solving all possible SDEs at once, since that will guarantee satisfaction of the Ward identities. However, it is possible to find approximate three- and higher-point proper vertices that satisfy the Ward identities exactly and that are expressed solely in terms of the PT propagator itself. This approximation, known as the gauge technique, is valid for infrared-dominated phenomena. Although we will not give any details here, we have in mind the gauge technique of Ref. [6], which gives the following expression (group indices suppressed) for the gauge technique proper vertex:

$$
\hat{\Gamma}_{\alpha \beta \gamma}\left(k_{1}, k_{2}, k_{3}\right)=g_{\alpha \beta}\left(k_{1}-k_{2}\right)_{\gamma}-\frac{k_{1 \alpha} k_{2 \beta}}{2 k_{1}^{2} k_{2}^{2}}\left(k_{1}-k_{2}\right)^{\mu} \hat{\Pi}_{\mu \gamma}\left(k_{3}\right)-\left[P_{\alpha}^{\mu}\left(k_{1}\right) \hat{\Pi}_{\mu \beta}\left(k_{2}\right)-\hat{\Pi}_{\alpha}^{\mu}\left(k_{1}\right) P_{\mu \beta}\left(k_{2}\right)\right] \frac{k_{3 \gamma}}{k_{3}^{2}}+c y c . p e r m .
$$

where the first term on the right is the bare vertex $\hat{\Gamma}^{0}$. Here $\hat{\Pi}_{\mu \nu}=P_{\mu \nu} \hat{\Pi}$ is the PT proper self-energy introduced above. This vertex satisfies:

$$
k_{1}^{\alpha}\left\{\hat{\Gamma}_{\alpha \beta \gamma}\left(k_{1}, k_{2}, k_{3}\right)-\hat{\Gamma}_{\alpha \beta \gamma}^{0}\left(k_{1}, k_{2}, k_{3}\right)\right\}=\hat{\Pi}_{\beta \gamma}\left(k_{2}\right)-\hat{\Pi}_{\beta \gamma}\left(k_{3}\right)
$$

no matter what the choice of $\hat{\Pi}$ is. In consequence, an approximate but fully gauge-invariant one-dressed-loop SDE for the PT propagator can be written solely in terms of that propagator. It is in this sense that we speak of studying the PT propagator on its own terms, without further specification of the vertex beyond that of the gauge technique. In this paper we use the self-contained PT propagator equation of Ref. [6]. Later we will study a toy model of a "half-proper" three-gluon PT vertex SDE in which some factors coming from the propagators are incorporated in the definition of the vertex, and we arrive at essentially self-contained vertex SDEs. 


\section{B. Sign problems}

Define a K-L function as a real-analytic function with at most one pole and a cut along the real positive axis, satisfying an unsubtracted dispersion relation with a positive spectral function.

We conjecture that the PT propagator is the product of two K-L functions. The product of two K-L functions may or may not be a K-L function, but in the present case we know it cannot be, because the product vanishes faster than $1 / q^{2}$ near infinity. The product of two K-L functions having this property therefore requires a spectral function that changes sign. Multiply together two K-L functions, call them $G_{1}$ and $G_{2}$. For each function we have:

$$
G_{i}=\int d \sigma \frac{\rho_{i}(\sigma)}{q^{2}-\sigma}(i=1,2)
$$

with $\rho_{i}$ nowhere negative. The product $G_{1} G_{2}$ obeys the dispersion relation:

$$
G_{1} G_{2}=\int d \sigma \frac{\rho_{1 \times 2}(\sigma)}{q^{2}-\sigma}
$$

with

$$
\rho_{1 \times 2}(\sigma)=\mathcal{P} \int d \sigma^{\prime} \frac{\rho_{1}(\sigma) \rho_{2}\left(\sigma^{\prime}\right)+\rho_{1}\left(\sigma^{\prime}\right) \rho_{2}(\sigma)}{\sigma-\sigma^{\prime}}
$$

which may be negative in places.

We note parenthetically that it is possible, in certain field theories involving scalar particles, for the propagator (taken to be K-L) to have a single zero between the particle mass and the lowest two-particle threshhold and for the proper vertex to have a pole at the same place. In our case this would correspond to a zero in $\hat{H}\left(q^{2}\right)$ for $m^{2}<q^{2}<4 m^{2}$. But for propagator models what we use as a criterion for a critical mass, based on asymptotic freedom, is not to exclude a zero of the propagator but to exclude an unwanted pole. It is true that the vertex models we study define a "critical" mass by excluding vertex singularities, but these are not related in any obvious way to zeroes of $\hat{H}$. In any case we explicitly exclude the possibility of a zero in $\hat{H}$ by assumption, and our techniques show no signs of such a zero developing. See [39] for further details and references.

\section{Mass parametrization}

The conventional approach to the propagator, whether from the PT or elsewhere, is to calculate in some approximation the proper self-energy, that is, the inverse propagator. The technique of going from the propagator to the inverse propagator (or vice versa) reminds us of analytic perturbation theory (APT), which we discuss in the following section. APT was used in the NAGT context to render $\alpha_{s}(0)$ finite, even in the zero-mass limit. However, an unphysical singularity remains, and in fact mass-improved APT also has a critical mass $m_{c}$ that is rather close to the values we give in this section.

We assume that there is at most one pole in the PT propagator, at $q^{2}=m^{2}$, representing dynamical gluon mass formation, and no zeroes, and we replace the factor $\hat{H}$ of Eq. (4) by the simple massive propagator $\left(q^{2}-m^{2}+i \epsilon\right)^{-1}$, where the mass does not run. Our pretensions to accuracy in the infrared do not justify saving the running, so $m^{2}$ can be thought of as either the running mass at zero momentum or the pole mass, within the accuracy to which we aspire.

Long ago, a form of the pinch technique was used to estimate the dynamical gluon mass [1, 6], and interpreted in the factorized form outlined above. In the formulas of [ 6 ] we ignore the running of the mass, and the non-linear integral equation then becomes:

$$
\left[g^{2} \hat{d}(q)\right]^{-1}=q^{2} b Z-\frac{i b}{\pi^{2}} \int d^{4} k \hat{H}(k) \hat{H}(k+q)\left\{q^{2}+\frac{m^{2}}{11}\right\}+C+\ldots
$$

where the constant $C$ summarizes the seagull graph and other momentum-independent terms, and we also omit twoloop contributions. Rather than trying to solve this equation we simply replace $\hat{H}$ by a free massive propagator, yielding:

$$
\left[g^{2} \hat{d}\left(q^{2}\right)\right]^{-1}=q^{2} b Z+b J\left(q^{2} ; \Lambda_{U V}^{2}\right)\left\{q^{2}+\frac{m^{2}}{11}\right\}+C+\ldots
$$


where $\Lambda_{U V}$ is an ultraviolet cutoff. Omitted terms are of higher order in a dressed-loop expansion. The integral $J\left(q^{2} ; \Lambda_{U V}^{2}\right)$ is:

$$
J\left(q^{2} ; \Lambda_{U V}^{2}\right)=\frac{i}{\pi^{2}} \int d^{4} k \frac{1}{\left(k^{2}-m^{2}+i \epsilon\right)\left((k-q)^{2}-m^{2}+i \epsilon\right)} ;
$$

and the UV cutoff is defined through the Feynman-parameter representation:

$$
J\left(q^{2} ; \Lambda_{U V}^{2}\right)=\int_{0}^{1} d \alpha \ln \left\{\frac{m^{2}-\alpha(1-\alpha) q^{2}-i \epsilon}{\Lambda_{U V}^{2}}\right\} .
$$

By appropriate choice of $Z$ we make the combination $Z+J$ finite, and define a renormalized integral $J\left(q^{2} ; \xi\right)$. Aside from its Feynman-parameter form $J$ has a dispersive representation:

$$
J\left(q^{2} ; \xi\right)=\int_{0}^{1} d \alpha \ln \left\{\frac{m^{2}-\alpha(1-\alpha) q^{2}-i \epsilon}{\xi}\right\}=-q^{2} \int_{4 m^{2}}^{\infty} \frac{d \sigma}{\sigma} \sqrt{1-\frac{4 m^{2}}{\sigma}} \frac{1}{\sigma-q^{2}-i \epsilon}+\ln \left(\frac{m^{2}}{\xi}\right) .
$$

The PT inverse propagator in finite terms is:

$$
\left[g^{2} \hat{d}\left(q^{2}\right)\right]^{-1}=q^{2} b \tilde{Z}+b\left(q^{2}+\frac{m^{2}}{11}\right) J\left(q^{2} ; \xi\right)+C+\ldots
$$

Note that this is consistent with the renormalization-invariance of Eq. (1). We make a choice of $\xi$ (or $\tilde{Z}$ ) that defines what we mean by $\Lambda$, the finite QCD scale, by requiring that

$$
\hat{d}^{-1}\left(q^{2}\right) \rightarrow b g^{2} q^{2} \ln \left(\frac{-q^{2}}{\Lambda^{2}}\right)[1+o(1)]
$$

as $q^{2}$ approaches infinity in any direction. (The non-leading terms are $\mathcal{O}\left(\ln \ln q^{2}\right)$ and can affect the definition of $\Lambda$ at any particular momentum, but since we deal here only with one-dressed-loop quantities we cannot use such higher-order terms in the analysis; one should think of $\Lambda$ as applying to a specific range of large but finite momenta and that effectively incorporates terms not vanishing at infinity.) We choose:

$$
\xi=e^{-2} \Lambda^{2}
$$

and then we can set $\tilde{Z}=0$.

Since the inverse propagator is to vanish at $q^{2}=m^{2}$ we can eliminate $C$ by writing:

$$
\left[g^{2} \hat{d}\left(q^{2}\right)\right]^{-1}=b\left\{J\left(q^{2} ; \xi\right)\left(q^{2}+\frac{m^{2}}{11}\right)-J\left(m^{2} ; \xi\right) \frac{12 m^{2}}{11}\right\} .
$$

This can be written in dispersive form by using Eq. (16):

$$
\left[g^{2} \hat{d}\left(q^{2}\right)\right]^{-1}=b\left(q^{2}-m^{2}\right)\left\{2+\ln \left(\frac{m^{2}}{\Lambda^{2}}\right)-\int_{4 m^{2}}^{\infty} d \sigma \sqrt{\frac{\sigma-4 m^{2}}{\sigma}} \frac{1}{\sigma-q^{2}-i \epsilon}\left[\frac{q^{2}}{\sigma}+\frac{12 m^{2}}{11\left(\sigma-m^{2}\right)}\right]\right\} .
$$

We now assume, as discussed in connection with Eq. (4), that

$$
g^{2} \hat{d}\left(q^{2}\right)=\frac{\bar{g}^{2}\left(q^{2}\right)}{q^{2}-m^{2}+i \epsilon}
$$

which yields:

$$
\left[b \bar{g}^{2}\left(q^{2}\right)\right]^{-1}=2+\ln \left(\frac{m^{2}}{\Lambda^{2}}\right)-\int_{4 m^{2}}^{\infty} d \sigma \sqrt{\frac{\sigma-4 m^{2}}{\sigma}} \frac{1}{\sigma-q^{2}-i \epsilon}\left[\frac{q^{2}}{\sigma}+\frac{12 m^{2}}{11\left(\sigma-m^{2}\right)}\right] .
$$

Provided that $m / \Lambda$ is sufficiently large, a condition that we will investigate below and assume for now, the dispersion relation for the propagator has the properties discussed in Sec. IIB above. We write:

$$
\bar{g}^{2}\left(q^{2}\right)=\frac{1}{\pi} \int_{4 m^{2}}^{\infty} d \sigma \frac{\rho(\sigma)}{\sigma-q^{2}-i \epsilon}
$$


with $\rho$ a function easily read off from Eq. (23); we need not record it explicitly. This equation shows that $\rho(\sigma)$ is positive, as we expect. An elementary calculation shows that the dispersion relation for $-g^{2} \hat{d}\left(q^{2}\right)$, as taken from Eq. (22), is:

$$
-g^{2} \hat{d}\left(q^{2}\right)=\frac{R}{m^{2}-q^{2}-i \epsilon}-\frac{1}{\pi} \int d \sigma \frac{\rho(\sigma)}{\left(\sigma-m^{2}\right)\left(\sigma-q^{2}-i \epsilon\right)}
$$

where $R$ is a positive residue, the on-shell value of $\bar{g}^{2}$ :

$$
R=\frac{1}{\pi} \int d \sigma \frac{\rho(\sigma)}{\sigma-m^{2}} .
$$

Although each of the two factors $\bar{g}^{2}\left(q^{2}\right)$ and $1 /\left(m^{2}-q^{2}\right)$ have positive imaginary parts, the imaginary part of their product, which is:

$$
\operatorname{Im}\left[-g^{2} \hat{d}(\sigma)\right]=R \delta\left(\sigma-m^{2}\right)-\frac{\rho(\sigma)}{\sigma-m^{2}}
$$

has one positive term from the pole and another term from the cut that is everywhere negative. This is, of course, required by the large- $q$ behavior of the product which requires that the integral of the imaginary part vanish.

One implication of the dispersive form in Eq. (23) is that $\bar{g}^{2}$ is positive everywhere where it is real, that is, in the region $-\infty<q^{2}<4 m^{2}$. Another is that $\bar{g}^{2}$ is monotonically increasing as $q^{2}$ decreases. Since we expect $\bar{g}^{2}$ to be monotonically decreasing as the mass increases, there is a critical mass $m_{c}$ such that if the physical mass exceeds $m_{c}$ there is no spurious pole, while there is such a pole if $m<m_{c}$. This critical mass is determined by positivity of the running coupling just below the threshhold and yields $m_{c} / \Lambda=\exp [\sqrt{3} \pi / 33]=1.18$.

All the integrals in Eq. (23) can be evaluated, giving the running charge explicitly. In the regime $0<q^{2}<4 m^{2}$ the explicit result is:

$$
\begin{aligned}
{\left[b \bar{g}^{2}\left(q^{2}\right)\right]^{-1} } & =\frac{1}{q^{2}-m^{2}+i \epsilon}\left\{\left[q^{2}+\frac{m^{2}}{11}\right] J\left(q^{2} ; \xi\right)-\frac{m^{2}}{12} J\left(m^{2} ; \xi\right)\right\} \\
& =\ln \left(\frac{m^{2}}{\Lambda^{2}}\right)+\frac{2}{q^{2}-m^{2}}\left\{\left(q^{2}+\frac{m^{2}}{11}\right) \sqrt{\frac{4 m^{2}-q^{2}}{q^{2}}} \arctan \sqrt{\frac{q^{2}}{4 m^{2}-q^{2}}}-\frac{2 \sqrt{3} \pi}{11}\right\}
\end{aligned}
$$

which has an obvious analytic continuation to other regimes.

Let us compare this result for the running charge to the old ansatz of [1], which is:

$$
\left[b \bar{g}\left(q^{2}\right)\right]^{-1}=\ln \left[\left(4 m^{2}-q^{2}-i \epsilon\right) / \Lambda^{2}\right] .
$$

It is clear that this is not accurate for the above-threshhold region $q^{2} \geq 4 m^{2}$, because it has a pole at $q^{2}=4 m^{2}-\Lambda^{2}$, which is timelike, provided that $m>\Lambda / 2$, and lies below threshhold in the region where the running charge is real. However, this expression does not differ very much from the improved PT value of Eq. (28) above in the spacelike regime. In Fig. [1 we compare the old expression of Eq. (29) and the new expression in Eq. (23) plotted vs. $q^{2} / \mathrm{m}^{2}$, at a mass ratio $m / \Lambda=1$. For any other value, simply add $\ln \left(m^{2} / \Lambda^{2}\right)$ to both expressions. They differ by about $15 \%$ or less from their average in the spacelike regime but separate increasingly for $0<q^{2}<4 m^{2}$ as the 1982 expression approaches its timelike divergence.

For most phenomenological applications one is interested in the running charge at a small spacelike momentum transfer. Either the new expression from Eq. (23) or the old one from Eq. (29) shows that $\alpha_{s}(0)$ increases as $m$ decreases. A single formula applies to both cases, with one parameter $\rho$ whose value depends on whether the new or old expression is used. We find:

$$
\alpha_{s}(0)=\left\{\frac{1}{4 \pi b}\right\} \frac{1}{\ln \left(\frac{\rho^{2} m^{2}}{\Lambda^{2}}\right)}
$$

which is positive provided that $m / \Lambda \geq \rho^{-1}$. For the new expression:

$$
\rho_{\text {new }}^{-1}=\exp \left[-1+\frac{12}{11}\left(1-\frac{\sqrt{3} \pi}{6}\right)\right] \approx 0.41
$$

and for the old fit $\rho_{\text {old }}^{-1}=0.5$ - not much different. If $m / \Lambda \geq \rho^{-1}$ the squared running charge is positive for all spacelike $\left(q^{2}<0\right)$ momenta.

What happens when $m<m_{c}$ ? Is this unacceptable, or is there a fix through APT? 


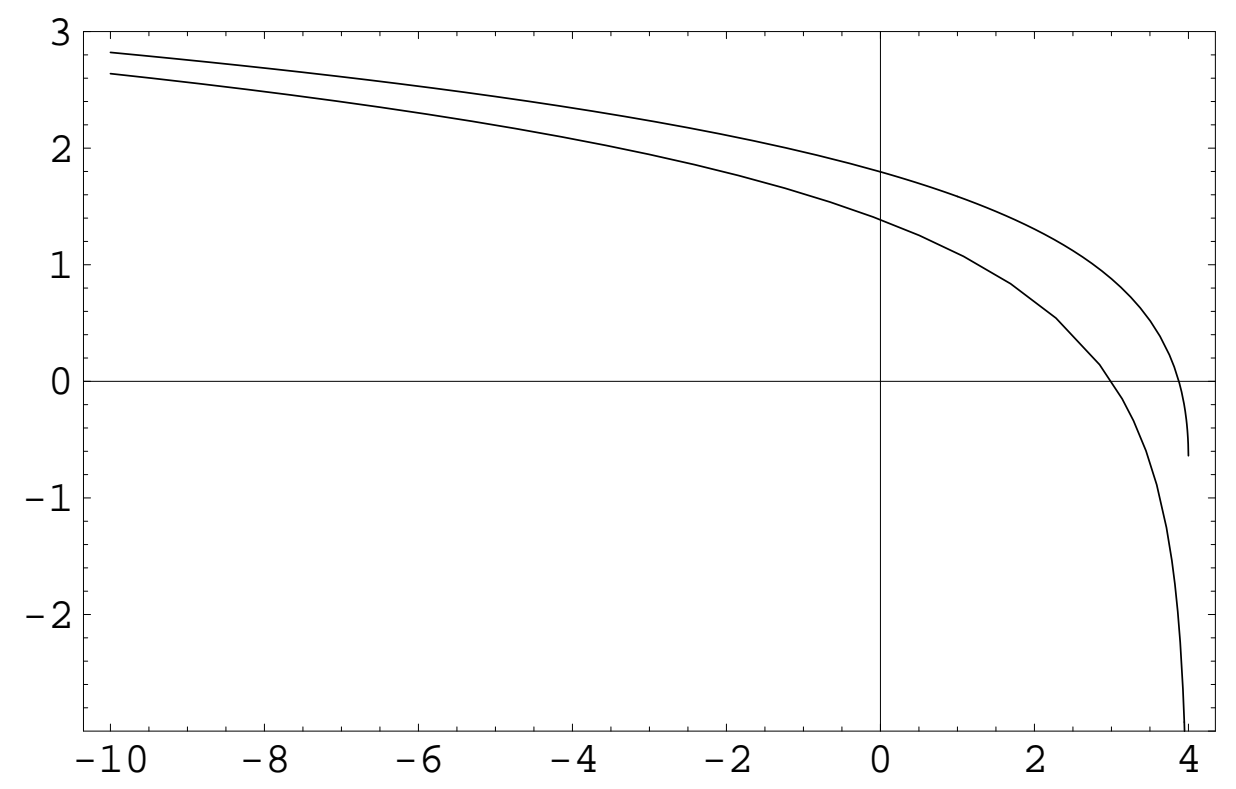

FIG. 1: Comparison of new and old expressions for $\left[b \bar{g}^{2}\left(q^{2}\right)\right]^{-1}$ below threshhold. The upper curve is the new [Eq. (23)] and the lower the old [Eq. (29)] expression at $m=\Lambda$; the $x$-axis is $q^{2} / m^{2}$.

\section{APT AND OTHER MODELS}

Massless APT [10] begins with ordinary perturbation theory for the running charge, which at one loop is:

$$
F\left(q^{2}\right) \equiv\left[\bar{g}\left(q^{2}\right)_{1}\right]^{-1}=b \ln \left(\frac{-q^{2}-i \epsilon}{\Lambda^{2}}\right) .
$$

The tachyonic pole at $q^{2}=-\Lambda^{2}$ is removed using a renormalization-group-improved extension of fifty-year-old techniques that impose correct analyticity properties on certain gauge-invariant quantities such as the Adler D-function or the photon propagator, which amounts to postulating the dispersion relation:

$$
\bar{g}^{2}\left(q^{2}\right)_{A P T}=\frac{1}{\pi} \int_{0}^{\infty} d \sigma \frac{-I m F}{|F|^{2}} \frac{1}{\sigma-q^{2}-i \epsilon} .
$$

As needed, the imaginary part of $\bar{g}_{A P T}^{2}$ is positive. Since the dispersion integral can have (by fiat) no pole, the simple result is:

$$
\alpha_{s, A P T}\left(q^{2}\right)=\frac{1}{4 \pi b}\left\{\frac{1}{\ln \left[\left(-q^{2}-i \epsilon\right) / \Lambda^{2}\right]}+\frac{\Lambda^{2}}{\Lambda^{2}+q^{2}}\right\}
$$

with a zero-momentum value of $\alpha_{s}(0)=1 /(4 \pi b) \simeq 1.4$ (for three-flavor QCD). Higher-order renormalization-group improvement changes this value only slightly. While this value for $\alpha_{s}(0)$ is certainly in the right ballpark, there is an uncomfortable flaw in APT. It predicts that the slope $d \alpha_{s}\left(q^{2}\right) / d q^{2}$ at $q^{2}=0$ is negative infinity, which is certainly unphysical. Consequently the predicted value in Eq. (34) is not reliable, although that is not our main concern here.

Obviously this massless APT treatment can be trivially extended to the old proposal of Eq. (29), but this just transfers the infinite slope to threshhold. This is a fault to be associated with the proposed running charge, which is simply not physical near threshhold.

Shirkov later [11] proposed to put in, by hand, a gluon mass in a different way that actually is close in spirit to the version of the PT we use here. Although Shirkov's work is unclear on some relatively insignificant details, when quarks are omitted it is essentially equivalent to the following expression:

$$
\left[b \bar{g}^{2}\left(q^{2}\right)\right]^{-1}=J\left(q^{2} ; \xi\right) .
$$

Just as in perturbation theory, $J\left(q^{2} ; \xi\right)$ may have an unphysical pole coming from a zero of $J$; in the massive case with $m / \Lambda>2$ this pole lies in the region $\Lambda^{2}<q^{2}<4 m^{2}$. The locus of zeroes in $J\left(q^{2} ; \xi\right)$ in Eq. (16) is:

$$
\ln \left(\frac{4 m^{2}}{\xi}\right)=2\left\{1-\gamma^{-1} \arctan \gamma\right\}
$$


where

$$
\gamma=\sqrt{\frac{q^{2}}{4 m^{2}-q^{2}}} .
$$

This yields $m_{c} \Lambda=1$. Of course, it might be possible to remove this singularity for $m<m_{c}$ by the same techniques used for massless APT, with the dispersion relation:

$$
b \bar{g}^{2}\left(q^{2}\right)=\frac{-1}{\pi} \int_{4 m^{2}}^{\infty} d \sigma \frac{\operatorname{Im} J(\sigma ; \xi)}{|J(\sigma ; \xi)|^{2}\left(\sigma-q^{2}-i \epsilon\right)} .
$$

However, from the expression for $J$ above threshhold:

$$
J\left(q^{2} ; \xi\right)=\ln \left(\frac{m^{2}}{\Lambda^{2}}\right)+\gamma^{-1} \ln \left\{\frac{\gamma+1}{\gamma-1}\right\}-i \pi \gamma^{-1}
$$

one sees that at the critical mass both the real and the imaginary parts of $J$ vanish at threshhold, leading to a singular running charge at threshhold. Presumably this is unphysical. There are no singularities for larger values of $m / \Lambda$, so it appears that for mass-improved APT there is a critical mass: $m_{c} / \Lambda=1$.

\section{TOY VERTEX MODELS AND POSITIVITY}

It is much too difficult to consider the full Schwinger-Dyson equations even at the lowest loop level for NAGTs, so we construct an analytically-soluble toy model. This new model is in the spirit of the old toy model of Ref. 13], which is not analytically-soluble. Both models have the same large-momentum behavior, showing asymptotic freedom and a beta-function with all terms negative and with factorial growth.

Both models exploit the fact that, just as the product $g^{2} \hat{d}\left(q^{2}\right)$ is not only gauge-invariant but renormalization-group invariant, there is a similar combination for the PT vertex. Introduce the notation:

$$
\hat{d}\left(q^{2}\right)=\hat{H}\left(q^{2}\right) \hat{Z}^{-1}\left(q^{2}\right) .
$$

With our factorization conjecture for the PT propagator this is equivalent to:

$$
\bar{g}^{2}\left(q^{2}\right)=\frac{g^{2}}{\hat{Z}\left(q^{2}\right)} .
$$

Because $\hat{g}^{2}\left(q^{2}\right)$ is positive for spacelike (negative) $q^{2}$, so is $\hat{Z}\left(q^{2}\right)$. Call the proper vertex function in the pinch technique $\hat{\Gamma}_{\mu \nu \alpha}^{a b c}\left(q_{1}, q_{2}, q_{3}\right)$. This vertex function, which like the PT propagator is gauge-invariant and process-independent, obeys a Ward identity of QED type, with no contributions from ghosts, schematically of the form $q_{1} \cdot \Gamma=\hat{Z}\left(q_{2}\right)-\hat{Z}\left(q_{3}\right)$. The gauge-invariant and renormalization-group invariant we call $\hat{G}$ (irrelevant group and spin indices omitted):

$$
\hat{G}\left(q_{1}, q_{2}, q_{3}\right)=\frac{g \hat{\Gamma}\left(q_{1}, q_{2}, q_{3}\right)}{\left(\hat{Z}\left(q_{1}\right) \hat{Z}\left(q_{2}\right) \hat{Z}\left(q_{3}\right)\right)^{1 / 2}}
$$

where $\hat{\Gamma}$ is the PT proper vertex function (again, irrelevant indices omitted) and $\hat{Z}$ is a factor in the propagator, as given in Eqs. (40[41). When all the momenta are $\mathcal{O}(q)$ the Ward identity tells us that $\hat{\Gamma} \sim g^{2} \bar{g}^{-2}$ at large momentum, and then Eq. (42) shows that $\hat{G} \sim \bar{g}(q)$, as would be expected for a gauge-invariant vertex function.

One might think that the PT Schwinger-Dyson equation (SDE) for $\hat{\Gamma}$ explicitly involves the PT propagator $\hat{d}$ that has a non-positive imaginary part. Instead we remark that this equation can be rewritten in terms only of the normal propagators $\hat{H}$ and the special vertex $\hat{G}$. This is important because $\hat{d}\left(q^{2}\right)$ itself violates the K-L representation, and if the skeleton graphs of the SDE were to be modeled by replacing bare propagators by $\hat{d}$ there could possibly be positivity problems in the SDE arising from the $\hat{d}$ terms. Schematically the one-dressed-loop SDE is:

$$
\hat{G}=\hat{G}_{0}+\int \hat{G}^{3} \hat{H}^{3}+\ldots
$$

Here $\hat{G}$ is the Born term, behaving like $\left(\ln q^{2}\right)^{-3 / 2}$ when all momenta are large and $\mathcal{O}(q)$. Note that this equation is independent of the coupling constant $g$, as it must be if $\hat{G}$ is renormalization-invariant; this independence holds for 
all vertex skeleton graphs. We can now draw conclusions based on the (Euclidean) positivity of the $\hat{H}$ propagators without fear of difficulties arising from non-positivity of the spectral function for the propagator itself.

So far we have not considered numerator factors. Neither toy model has them, but each roughly accounts for them by dropping one of the propagator factors in Eq. (43). We know that $\hat{G} \sim\left(\ln q^{2}\right)^{-1 / 2}$ at large $q$, which dominates over the inhomogeneous Born term. Consequently, in the toy model this inhomogeneous term is dropped. Furthermore in the toy model, $\hat{G}$ depends on only one momentum, and only the one-loop skeleton graph is saved. The original toy model equation [13] is then:

$$
\hat{G}(q)=\frac{i b}{2 \pi^{2}} \int \frac{d^{4} k}{\left(k^{2}-m^{2}+i \epsilon\right)\left[(q+k)^{2}-m^{2}+i \epsilon\right]} \hat{G}^{3}(k)
$$

where $b$ is the usual (no-quark) one-loop coefficient in the beta-function. This is a universal equation for any coefficient $b$ in the beta-function, as one sees by using the vertex $R \equiv b^{1 / 2} \hat{G}$ in place of $\hat{G}$.

We will work in Euclidean space, defining $Q^{2} \equiv-q^{2}$ as the Euclidean square of the momentum, positive for spacelike $q$. Then the kernel of the non-linear integral equation (44) is positive. If $m \neq 0$ the kernel is nowhere singular, but if $m=0$ the kernel is singular at zero momentum. One implication is that the massless $\hat{G}$ is necessarily zero or singular at zero momentum, already suggesting the necessity of a mass. We briefly review these facts for the massless model.

\section{A. Massless toy model}

The massless toy model can be converted [13] to a differential equation:

$$
\hat{G}_{t t}+\hat{G}_{t}=-\frac{b}{2} \hat{G}^{3}
$$

where the subscripts indicate derivatives with respect to the variable $t \equiv \ln \left(Q^{2} / \Lambda^{2}\right)$ and $\Lambda$ is the usual QCD mass scale. We would like to impose physically-sensible boundary conditions at $Q^{2}=0$, or $t=-\infty$, but this is impossible: The massless version of Eq. (44) is singular at zero momentum unless $\hat{G}(0)=0$, which we forbid. Nevertheless the differential equation can be solved, showing features expected from perturbation theory, and we can impose boundary conditions at $t>0$ and study the ultraviolet behavior.

In the ultraviolet regimes one finds results familiar from the RG: The functional form of the asymptotic vertex is precisely that of the full NAGT except for the value of some numerical coefficients, and all signs agree with what is needed for asymptotic freedom. For large $t$ the second derivative term is non-leading; if dropped, the general solution to the first-order differential equation is:

$$
\frac{1}{\hat{G}^{2}(t)}-b t=\text { const }
$$

This coupling is singular, as massless perturbation theory must be. When the second derivative term is kept, a solution is generated which has all the same terms as the all-order perturbative running charge in true QCD, but with somewhat different coefficients. All these coefficients have the correct sign for an asymptotically-free theory.

There is also an interesting beta-function, governed by its own differential equation. This comes from the relation:

$$
\hat{G}_{t}=\frac{1}{2} \beta(\hat{G})
$$

plus the equation (45) for the vertex, and is:

$$
\beta(g)\left[1+\frac{1}{2} \frac{d \beta}{d g}\right]=-b g^{3} .
$$

It was shown [13] that this beta-function behaves like $-g \sum N !\left(b c g^{2}\right)^{N}$ for some positive constant $c$, qualitatively just the same as in any asymptotically-free NAGT, and that the beta-function solving Eq. (48) is singular at a finite upper critical coupling $g_{c}$.

\section{B. Massive toy model}

We give here a new toy model inspired by but differing slightly from the original massive toy model of Eq. (44). The new model is probably about as accurate as the original in modeling the true SDE, but it can be analytically 
solved and shows a critical mass value. It has the same ultraviolet (massless) properties as the old model and as QCD does itself. As before we take $\hat{H}$ as a free massive propagator. There is no longer a simple differential form of the original toy model equation Eq. (44) when masses are included, but the following approximation to the Euclidean angular integration does give an ordinary differential equation:

$$
\int \frac{d \Omega_{K}}{2 \pi^{2}} \frac{1}{\left[(Q+K)^{2}+m^{2}\right]} \approx \frac{\theta\left(Q^{2}-K^{2}\right)}{Q^{2}+m^{2}}+\frac{\theta\left(K^{2}-Q^{2}\right)}{K^{2}+m^{2}}
$$

The approximation is exact for large $Q, K$ as well as when $Q>0, K=0$ (or $K>0, Q=0$ ) and is otherwise too large by a factor which is at most about 1.3 times the true angular integral at $Q=K=m$. From this follows the one-dimensional integral equation:

$$
\begin{aligned}
\hat{G}\left(Q^{2}\right) & =J_{1}\left(Q^{2}\right)+J_{2}\left(Q^{2}\right) \\
J_{1}\left(Q^{2}\right) & =\frac{b}{2\left(Q^{2}+m^{2}\right)} \int_{0}^{Q^{2}} d K^{2} \frac{K^{2} \hat{G}^{3}\left(K^{2}\right)}{K^{2}+m^{2}} \\
J_{2}\left(Q^{2}\right) & =\frac{b}{2} \int_{Q^{2}}^{\infty} d K^{2} \frac{K^{2} \hat{G}^{3}\left(K^{2}\right)}{\left(K^{2}+m^{2}\right)^{2}}
\end{aligned}
$$

Every solution of this integral equation $\hat{G}$ satisfies the differential equation:

$$
\left[\left(Q^{2}+m^{2}\right) \hat{G}\left(Q^{2}\right)\right]^{\prime \prime}=-\frac{b Q^{2} \hat{G}^{3}\left(Q^{2}\right)}{2\left(Q^{2}+m^{2}\right)^{2}}
$$

where a prime indicates differentiation with respect to $Q^{2}$. If we now define $t$ as:

$$
t=\ln \left(\frac{Q^{2}+m^{2}}{\Lambda^{2}}\right)
$$

the differential equation can be written as an extension of the massless equation:

$$
\hat{G}_{t t}+\hat{G}_{t}=-\frac{b}{2} \hat{G}^{3}\left(1-\frac{m^{2} e^{-t}}{\Lambda^{2}}\right) .
$$

The difference from the massless equation is that at $Q=0$ the variable $t$ is finite, not $-\infty$.

It is not difficult to check from the integral equation (50) that at large momenta only the $J_{2}$ term is leading, yielding the exact leading behavior $\hat{G}\left(Q^{2}\right) \rightarrow 1 /\left(b \ln Q^{2}\right)^{1 / 2}$ at large $Q^{2}$. The $J_{1}$ term in Eq. (50) is $\left.\mathcal{O}\left[\left(\ln Q^{2}\right)^{-3 / 2}\right)\right]$ and non-leading. In neither the original toy model nor at present are we interested in such non-leading terms, so we define our new toy model by dropping the $J_{1}$ term. Note also that $J_{1}=\mathcal{O}\left(Q^{4}\right)$ at small $Q^{2}$, while $J_{2}=\mathcal{O}(1)$, since we require that $\hat{G}(0) \neq 0$; the missing $J_{1}$ term is non-leading in the infrared as well. [One can show that the $\mathcal{O}\left(Q^{4}\right)$ terms in $J_{1}$ and $J_{2}$ cancel in the full equation, leaving corrections to $\hat{G}(0)$ of $\mathcal{O}\left(Q^{6}\right)$. In fact, the exact solution $\hat{G}$ of Eq. (50) with both terms show the self-consistent behavior leading correction at small momentum:

$$
\left.\hat{G}\left(Q^{2}\right) \simeq \hat{G}(0)-\frac{\hat{G}(0)^{3}}{12}\left(\frac{Q^{2}}{m^{2}}\right)^{3}+\ldots\right]
$$

Evidently the resulting integral equation with no $J_{1}$ term satisfies a first-order differential equation, which is just Eq. (54) without the $\hat{G}_{t t}$ term. This equation has the exact solution $\hat{G}_{1}$ :

$$
\frac{1}{\hat{G}_{1}^{2}\left(Q^{2}\right)}=b\left[\ln \left(\frac{Q^{2}+m^{2}}{\Lambda^{2}}\right)+\frac{m^{2}}{Q^{2}+m^{2}}\right] .
$$

One can check that the beta-function coming from $\hat{G}_{1}$ has not only the usual $-b \hat{G}_{1}^{3}$ term but also terms involving non-perturbative quantities such as $\exp \left[-1 /\left(b \hat{G}_{1}^{2}\right)\right]$. This approximation already shows a mass $m_{0}$ at which $\alpha_{s}(0)$ is singular: $m_{0} / \Lambda=e^{-1 / 2} \approx 0.61$; smaller values lead to a pole in $\hat{G}_{1}$. (We have done a quick numerical study of the differential equation Eq. (54) of the modified model, and found $m_{0} / \Lambda \approx 0.66$.) The actual critical mass $m_{c}$ might be about twice as large as $m_{0}$, based on our experience with the propagator model. 


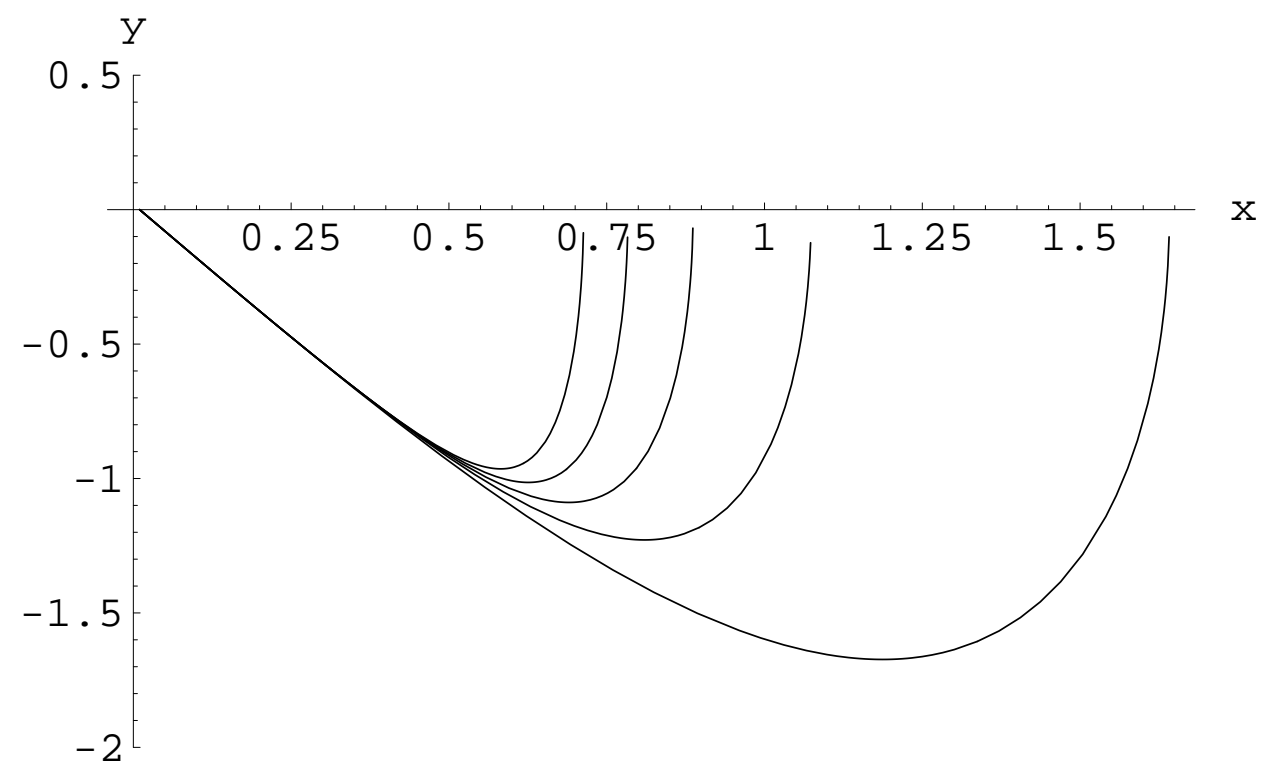

FIG. 2: Solutions to the differential equation (57) for $K=1$ (right-most curve) to $K=5$ (left-most curve). Here $x=\sqrt{b} g$ and $y=\sqrt{b} \beta$.

TABLE I: Upper-bound couplings for various $K$

\begin{tabular}{|l||l|l|l|l|l|}
\hline$K=$ & 1 & 2 & 3 & 4 & 5 \\
\hline$\alpha_{c}=$ & 3.1 & 1.3 & 0.90 & 0.70 & 0.58 \\
\hline
\end{tabular}

\section{Is a gluon mass really necessary?}

One may ask whether higher-order effects in a massless theory can somehow do away with the need for a gluon mass. To study this possibility, let us now extend the basic one-loop beta-function equation (48) to mimic multi-loop vertex contributions. Of course, we can only aspire to qualitative accuracy, looking for mechanisms of limits on $g^{2}$ rather than for accurate values of these limits. These models are essentially those of [40] devised for $\phi_{6}^{3}$, with coupling $G$. In this theory it has been shown that the sum of all $K$-loop vertex graphs is always positive and grows like $K !\left(c G^{2}\right)^{K}$, where $c$ is a positive number. We conjecture that the same holds for $d=4$ NAGTs, and that an appropriate extension of the beta-function differential equation to higher loops is:

$$
y\left(1+\frac{1}{2} y^{\prime}\right)=-x \sum_{J=1}^{K} J ! x^{2 J}
$$

where

$$
x=\sqrt{b} g, y(x)=\sqrt{b} \beta(g)
$$

and the prime denotes an $x$ derivative. One can verify that the beta-function solving this equation grows factorially like $K !\left(c g^{2}\right)^{K}$ for some $c$, whose value is irrelevant to our investigation. Our only interest is in seeing what happens when the right-hand side of the vertex Schwinger-Dyson equation has terms that grow factorially with loop number $K$. In fact, we have tested the sensitivity of Eq. (57) to fairly major changes in the coefficients on the right-hand side, and find little change from the solutions to Eq. (57) as shown in Fig. 2 ,

Table 1 show the upper bounds $\alpha_{c}$ for quarkless QCD, defined as the value of $g_{c}^{2} /(4 \pi)$ at which the beta-function crosses the real axis, for various values of $K$. One should expect the $\alpha_{c}$ values to decrease as $K$ increases, since otherwise the right-hand side of the differential equation will grow too large to balance the left-hand side. For all values of $K$ there is a singularity of the form $\beta \sim\left(g_{c}-g\right)^{1 / 2}$, where $\beta$ has infinite slope at $g=g_{c}$ and then turns imaginary for $g>g_{c}$. Note that there seems to be numerical convergence toward a value near 0.5 , not far from "best" estimates based on the one-dressed-loop pinch technique with a mass. But there is always a singularity. 
Perhaps some way of summing the non-Borel-summable series of Eq. (57) would remove this singularity. We have tried, again in the spirit of [40], "regulating" the all-orders behavior with a principal part integral form of the Borel integral corresponding to the sum in Eq. (57), using:

$$
y\left(1+\frac{1}{2} y^{\prime}\right)=L(x) .
$$

with:

$$
L(x)=-\frac{x^{2}}{\pi^{1 / 2}} \int_{0}^{\infty} \frac{d \alpha}{\alpha^{1 / 2}}\left\{\frac{e^{-\alpha / x^{2}}-e^{-1 / x^{2}}}{1-\alpha}\right\} .
$$

The equation for the vertex itself, analogous to Eq. (45), is:

$$
\hat{G}_{t t}+\hat{G}_{t}=b^{-1 / 2} L\left(b^{1 / 2} \hat{G}\right) .
$$

Actually, some form of principal-part regulation is demanded by dynamical boson mass generation [40], but we need not inquire further into that here. We can understand the basic behavior of the equation by looking at the degree to which factorial growth at large coupling is tamed by the principal-part prescription. The power-series expansion of $L(x)$ is

$$
L(x)=-\frac{1}{\sqrt{\pi}} \sum_{K=1} x^{2 K+1} \Gamma\left(K-\frac{1}{2}\right)+\mathcal{O}\left(e^{-1 / x^{2}}\right)
$$

and its asymptotic behavior at infinity is $-2 x$. This is very different from the finite- $K$ models of Eq. (57). In these, the increasingly-strong growth with $x$ of the right-hand side as $K$ gets larger means that any singularity occur at smaller values of $x$. But for equations with the $L(x)$ source there is no such movement toward smaller couplings because $L(x)$ is not growing rapidly at large $x$. In the beta-function equation (59) one easily finds the large- $x$ asymptotic behavior $y(x) \rightarrow-c x$ with $c=-1+\sqrt{5}$. So instead of generating a finite- $g$ singularity, the beta-function turns from $-b g^{3}$ behavior near the origin to linear at large enough $g$; numerical simulations confirm this. For the massless vertex equation (61) there is still a singularity in the infrared, so that $\hat{G}$ gets large and the large $x$ behavior of $L(x)$ matters. In this regime the massless equation (61) becomes linear, and one finds unphysical behavior of the type $\left(\ln Q^{2}\right)^{-1 / 2} \cos \left(\sqrt{3} \ln Q^{2}\right)$. So massless vertex dynamics is not regularized by the specific behavior of $L(x)$. Mass is important not only for the right-hand sides of the vertex and beta-function equations, as summarized by the function $L$, but it is important in the left-hand side of such equations, as we show below. In fact, the mass damping is so strong that it is probably unnecessary to worry about vertex graphs of very high order; $\hat{G}$ does not get large enough to probe the asymptotic limit of $L\left(b \hat{G}^{2}\right)$, as it does for the massless case.

\section{SUMMARY AND CONCLUSIONS}

We argue that although the gauge-invariant PT propagator does not obey the K-L representation, it is the product of two factors that do have the required positive spectral functions. We show that this holds true for an analytic approximation to the one-dressed-loop PT propagator equation, and that this approximation implies a critical mass $m_{c}$ such that the true dynamical mass $m$ must exceed $m_{c}$ or spurious singularities arise. We construct an analyticallysoluble toy model of one-dressed-loop PT three-gluon vertex model with a cubic non-linearity and show how the positivity of the factor $\hat{H}$ in the PT propagator plays an essential role in the vertex dynamics, in particular the occurrence of a mass value which must be exceeded by $m$ to avoid unwanted singularities. We argue that higherorder, even regulated all-order, extensions of the massless toy model equations do not remove these singularities.

[1] J. M. Cornwall, Phys. Rev. D 26, 1453 (1982).

[2] J. E. Mandula and M. Ogilvie, Phys. Lett. B 185, 127 (1987).

[3] C. A. Aubin and M. C. Ogilvie, Phys. Lett. B 570, 59 (2003).

[4] P. O. Bowman et al., Phys. Rev. D 76, 094505 (2007).

[5] A. C. Aguilar, A. Mihara and A. A. Natale, Phys. Rev. D 65, 054011 (2002).

[6] J. M. Cornwall and W. S. Hou, Phys. Rev. D 34, 585 (1986).

[7] A. C. Aguilar, D. Binosi and J. Papavassiliou, PoS LC2008, 050 (2008). 
[8] A. C. Aguilar, D. Binosi and J. Papavassiliou, Phys. Rev. D 78, 025010 (2008).

[9] J. M. Cornwall, Phys. Rev. D 76, 025012 (2007).

[10] D. V. Shirkov and I. L. Solovtsov, Phys. Rev. Letters 79, 1209 (1997).

[11] D. V. Shirkov, Phys. Atom. Nucl. 62, 1928 (1999) [Yad. Fiz. 62, 2082 (1999)].

[12] A. V. Nesterenko and J. Papavassiliou, Int. J. Mod. Phys. A 20, 4622 (2005); Phys. Rev. D 71, 016009 (2005).

[13] J. M. Cornwall and J. Papavassiliou, Phys. Rev. D 40, 3474 (1989).

[14] D. Binosi and J. Papavassiliou, Phys. Rev. D 66, 111901 (2002); J. Phys. G 30, 203 (2004).

[15] L. F. Abbott, Nucl. Phys. B 185, 189 (1981).

[16] J. M. Cornwall, in "Deeper Pathways in High-Energy Physics", ed. B. Kursonoglu, A. Perlmutter, and L. Scott, p. 683 (Plenum, New York, 1977).

[17] V. L. Baltar, H. G. Dosch and E. Ferreira, arXiv:0907.5310 [hep-ph].

[18] D. Binosi and J. Papavassiliou, Phys. Rev. D 77, 061702 (2008); JHEP 0811, 063 (2008).

[19] M. Lavelle, Phys. Rev. D 44, R26 (1991).

[20] C. Alexandrou, P. de Forcrand and E. Follana, Phys. Rev. D 63, 094504 (2001).

[21] C. Alexandrou, P. de Forcrand and E. Follana, Phys. Rev. D 65, 114508 (2002).

[22] Ph. Boucaud et al., arXiv:hep-ph/0507104.

[23] P. O. Bowman, U. M. Heller, D. B. Leinweber, M. B. Parappilly and A. G. Williams, Phys. Rev. D 70, 034509 (2004).

[24] Ph. Boucaud et al., arXiv:hep-lat/0602006

[25] A. Cucchieri, T. Mendes, O. Oliveira and P. J. Silva, Phys. Rev. D 76, 114507 (2007).

[26] O. Oliveira and P. J. Silva, Eur. Phys. J. A 31, 790 (2007).

[27] I. L. Bogolubsky, E. M. Ilgenfritz, M. Müller-Preussker and A. Sternbeck, PoS LAT2007, 290 (2007).

[28] A. Sternbeck, L. von Smekal, D. B. Leinweber and A. G. Williams, PoS LAT2007, 340 (2007).

[29] I. L. Bogolubsky, V. G. Bornyakov, G. Burgio, E. M. Ilgenfritz, M. Müller-Preussker, P. Schemel and V. K. Mitrjushkin, PoS LAT2007, 318 (2007).

[30] O. Oliveira, P. J. Silva, E. M. Ilgenfritz and A. Sternbeck, PoS LAT2007, 323 (2007).

[31] P. O. Bowman et al., Phys. Rev. D 76, 094505 (2007).

[32] E. M. Ilgenfritz, M. Müller-Preussker, A. Sternbeck, A. Schiller and I. L. Bogolubsky, Braz. J. Phys. 37, 193 (2007).

[33] A. Cucchieri and T. Mendes, PoS LAT2007, 297 (2007).

[34] A. Cucchieri and T. Mendes, Phys. Rev. Lett. 100, 241601 (2008).

[35] Ph. Boucaud, J. P. Leroy, A. Le Yaouanc, J. Micheli, O. Pene and J. Rodriguez-Quintero, JHEP 0806, 099 (2008).

[36] Zhang, Y-B, J-L Ping, X-F Lu, and H-S Zong, Comm. Theor. Phys. (Beijing) 50, 125 (2008).

[37] M. Gong, Y. Chen, G. Meng and C. Liu, arXiv:0811.4635 [hep-lat], December, 2008.

[38] I. L. Bogolubsky, E. M. Ilgenfritz, M. Müller-Preussker and A. Sternbeck, arXiv:0901.0736 [hep-lat].

[39] S. D. Drell, A. C. Finn and A. C. Hearn, Phys. Rev. 136, B1439 (1964).

[40] J. M. Cornwall and D. A. Morris, Phys. Rev. D 52, 6074 (1995). 